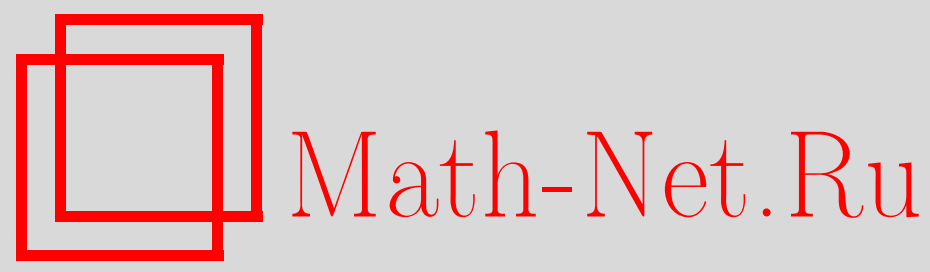

В. И. Сенашов, Характеризация групп с обобщенно черниковской периодической частью, Матем. заметки, 2000, том 67, выпуск 2, 270-275

DOI: https://doi.org/10.4213/mzm835

Использование Общероссийского математического портала Math-Net.Ru подразумевает, что вы прочитали и согласны с пользовательским соглашением http://www . mathnet.ru/rus/agreement

Параметры загрузки:

IP: 3.80 .181 .102

26 апреля 2023 г., 13:18:43

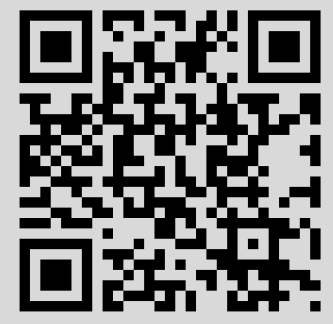




\section{ХАРАКТЕРИЗАЦИЯ ГРУПП С ОБОБЩЕННО ЧЕРНИКОВСКОЙ ПЕРИОДИЧЕСКОЙ ЧАСТЬЮ}

\section{В.И. Сенашов}

Получена характеризация группы с обобщенно черниковской периодической частью сначала в классе групп без элементов порядка 2 , а затем и без этого ограничения. Обобщаются на смешанные группы две теоремы автора для периодических групп.

Библиографоия: 16 названий.

1. Одним из основных методов исследования бесконечных групп является наложение на группу условия обрыва цепочек подгрупп. $\mathrm{K}$ таким условиям относятся условие примарной минимальности, условие минимальности для абелевых подгрупп и, наконец, просто условие минимальности для подгрупп. $\mathrm{K}$ другому виду условий конечности, не связанных с обрывом цепочек подгруп, относятся: бинарная конечность, бипримитивная конечность, сопряженно бипримитивная конечность. Эти условия касаются конечности тех или иных двупорожденных подгрупп в группе или ее сечениях.

Напомним, что группа $G$ называется сопряженно бипримитивно конечной, если для любого простого $p$ и для каждой конечной подгрупшы $H$ из $G$ любые два сопряженных элемента порядка $p$ из $N_{G}(H) / H$ порождают конечную подгруппу (определение введено В. П. Шунковым).

Связь между условиями конечности этих двух типов исследовалась С.Н. Черниковым, В.П. Шунковым, М.И. Каргаполовым и другими авторами.

2. В данной работе также удается связать условия указанных типов, а именно, доказать распространимость условия первого типа с системы подгрупп на всю группу (см. теоремы 1,2 ) при наложении на группу условия второго типа. При этом показывается, что условие второго типа существенно для формулировок теорем и его отбрасывание нарушает справедливость их утверждений.

В теореме 1 получают характеризацию групы с обобщенно черниковской периодической частью в классе групп без элементов порядка 2, а затем, в теореме 2 они охарактеризованы в классе всех групп на основе результата автора из [1].

Теоремы 1,2 обобщают на смешанные групшы теоремы 1,3 из [2].

Группа $G$ удовлетворяет условию примарной минимальности, если для любого простого $p$ каждая цепь

$$
G_{1}>G_{2}>\cdots>G_{n}>\cdots
$$

Работа выполнена при финансовой поддержке Красноярского краевого фонда науки, грант № $7 \mathrm{~F} 0008$. 
подгрупш из $G$ такая, что в любой разности $G_{n} \backslash G_{n+1}$ содержится элемент $g_{n}$ с условием $g_{n}^{p_{n}^{k_{n}}} \in G_{n+1}$ для некоторого $k_{n}$, обрьвается через конечное число шагов (определение принадлежит С.Н. Черникову).

Периодическая почти локально разрешимая группа, удовлетворяющая условию примарной минимальности, назьвается обобщенно черниковской группой.

Термин "обобщенно черниковские групп" впервые появился в [3]. Его использование можно обосновать тем, что по теореме Я. Д. Половицкого [4] обобщенно черниковская групша $G$ является расширением прямого произведения $A$ квазициклических $p$-групп с конечным числом множителей для каждого простого числа $p$ при помощи локально нормальной групшы $B$, причем каждьй элемент из $G$ поэлементно не перестановочен лишь с конечньм числом силовских примарных подгрупп из $A$. Для сравнения черниковская группа является конечньм расширением прямого произведения квазициклических групп, взятых в конечном числе.

Здесь будут доказаны две теоремы, характеризующие обобщенно черниковские группы сначала в классе групп без элементов порядка два (теорема 1), а затем без этого ограничения (теорема 2).

Теорема 1. Пусть $G$ - группа без әлементов второго порядка. Группа $G$ тогда и только тогда обладает обобщенно черниковской периодической частью, когда она сопря⿻енно бипримитивно конечна и нормализатор любой ее конечной нетривиальной подгруппы обладает обобщенно черниковской периодической частью.

Теорема 2. Группа тогда и только тогда обладает обобщенно черниковской периодической частью, когда она сопряженно бипримитивно конечна и нормализатор любой ее конечной нетривиальной подгруппь обладает обобщенно черниковской периодической частью.

Под периодической частьюгрупшы мы понимаем множество ее элементов конечного порядка, если последнее является группой.

Примеры групш С. П. Новикова, С.И. Адяна [5] и А. Ю. Ольшанского [6] показьвают, что условие сопряженно бипримитивной конечности в теоремах 1,2 существенно и его отбрасьвание приводит к потере справедливости утверждений теорем.

3. Перед доказательством теорем докажем несколько вспомогательных лемм. В них изучается сопряженно бипримитивно конечная група $G$ без элементов порядка два, в которой нормализатор $N_{G}(X)$ каждой нетривиальной конечной подгруппы $X$ из $G$ обладает обобщенно черниковской периодической частью.

Напомним некоторые необходимые определения.

Группа $G$ обладает полной частью $\widetilde{G}$, если $\widetilde{G}$ - абелева группа, порожденная всеми полными абелевьми подгруппами из $G$, и $G / \widetilde{G}$ не обладает полньми абелевыми подгруппами.

Элемент второго порядка называется инволюиией.

ЛЕмма 1. Силовские примарные подәруппы из $G$ черниковские.

ДокАЗАТЕЛьСтво. Ясно, что все примарные абелевы подгрупшы из $G$ удовлетворяют условию минимальности для подгрупп. Тогда каждая силовская подгрупша из $G$ удовлетворяет условию минимальности для абелевых подгрупп и поэтому является черниковской группой по теореме Остыловского из [7]. Лемма доказана. 
Лемма 2. Любая локально конечная подгруппа $B$ из $G$ обобщенно черниковская.

ДокАЗАТЕЛьСтво. Подгруппа $B$ локально разрешима по знаменитой теореме Файта-Томпсона. Так как силовские подгруппы из $G$ черниковские по лемме 1 , по теореме Каргаполова [8] в этом случае периодическая локально разрешимая группа $B$ обладает полной частью $A$. Если $A \neq 1$, то ясно, что $A$ обладает конечной нетривиальной характеристической подгруппой, и тогда $B$ - обобщенно черниковская группа по предположению.

Предположим теперь, что $A=1$. Из теоремы из [4] следует, что каждая обобщенно черниковская подгрупша из $B$ слойно конечна. В частности, нормализатор каждой конечной нетривиальной подгруппы из $B$ слойно конечен, и поэтому по теореме 2 из [9] $B$ сама слойно конечна. Таким образом, $B$ содержится в нормализаторе некоторой конечной нетривиальной подгрупшы и поэтому является обобщенно черниковской группой. Лемма доказана.

ЛЕмма 3. Если локально конечный радикал группы $G$ неединичен, то әруппа $G$ обладает обобщенно черниковской периодической частью.

ДокАЗАТЕЛЬСТво. Предположим, что локально конечньй радикал $L$ группы $G$ неединичен. Тогда $L$ - обобщенно черниковская группа по лемме 2 . Из слойной конечности локально нормальной подгруппы с черниковскими силовскими подгруппами (теорема Черникова, см., например, [10]) и по теореме из [4] следует, что либо $L$ обладает нетривиальной полной частью или она слойно конечна. В обоих случаях $L$ содержит конечную нетривиальную характеристическую подгруппу и, следовательно, по предположению $G$ обладает обобщенно черниковской периодической частью. Лемма доказана.

ЛЕмма 4. Любая обобщенно черниковская подгруппа $C$ из $G$ содержится в максимальной обобщенно черниковской подгруппе из $G$.

ДокАЗАТЕЛЬСТво. В виду упомянутой в п. 1 теоремы Половицкого обобщенно черниковская подгруппа $C$ локально конечна. По лемме Цорна она содержится в максимальной локально конечной подгруппе $V$ из $G$. Подгруппа $V$ является обобщенно черниковской по лемме 2. Лемма доказана.

ЛЕмма 5. Пересечение двух различных максимальных обобщенно черниковских подгрупп из $G$ конечно.

ДокАЗАТЕЛЬСтво. Предположим, что $A$ и $B$ - различные максимальные обобщенно черниковские подгрупшы из $G$ такие, что пересечение $D=A \cap B$ бесконечно.

Предположим сначала, что полная часть $\widetilde{D}$ групш $D$ нетривиальна. Тогда по предположению $N_{G}(\widetilde{D})$ обладает обобшенно черниковской периодической частью $H$ (обобщенно черниковская группа $\widetilde{D}$ обладает конечной характеристической подгрупой). Так как $\widetilde{A}$ и $\widetilde{B}$ абелевы и $\widetilde{D} \leqslant \widetilde{A} \cap \widetilde{B}$, то $\widetilde{A}$ и $\widetilde{B}$ содержатся в $H$; следовательно, они содержатся в $\widetilde{H}$. Так как обобщенно черниковская група $\widetilde{A}$ обладает конечной характеристической подгруппой, по предложению $N_{G}(\widetilde{A})$ обладает обобщенно черниковской периодической частью $A$ ( $A$ - максимальная обобщенно черниковская подгруппа); значит, $\widetilde{B} \leqslant \widetilde{H} \leqslant \widetilde{A}$. Точно так же мы получаем, что $\widetilde{A}$ содержится в $\widetilde{B}$, а значит, $\widetilde{A}=\widetilde{B}$ и, следовательно, $A$ совпадает с периодической частью $B$ группы (груша $N_{G}(\widetilde{B})$ обладает обобщенно черниковской периодической частью по той же причине, что и группа $\left.N_{G}(\widetilde{A})\right)$. Это противоречие показьвает, что $\widetilde{D}=1$ и, в частности, то, что група $D$ слойно конечна. 
Предположим теперь, что $A$ и $B$ обе обладают неединичньми полньми частями, и пусть $P$ и $Q$ - неединичные силовские $p$ - и $q$-подгруппы из $\widetilde{A}$ и $\widetilde{B}$ по некоторьп простым $p$ и $q$ соответственно. Так как $P$ - черниковская абелева нормальная подгруппа из $A$, фактор-группа $A / C_{A}(P)$ конечна по теореме Черникова как подгруппа периодической группы автоморфизмов полной абелевой черниковской групш; значит, в частности, $C_{D}(P)$ имеет конечный индекс в $D$. Аналогично, подгрупша $C_{D}(Q)$ имеет конечный индекс в $D$ и, следовательно, группа $M=C_{D}(P) \cap C_{D}(Q)$ бесконечна. Более того, $M$ слойно конечна, а значит, она обладает конечной нетривиальной характеристической подгруппой и $N_{G}(M)$ обладает обобщенно черниковской периодической частью $N$. Ясно, что $P$ содержится в $A \cap N$ и $N$ содержится в максимальной обобщенно черниковской подгрупе из $G$ по лемме 4 ; следовательно, как и в первой части доказательства, получаем $N \leqslant A$. Таким образом, $Q \leqslant N \leqslant A$. Это противоречие показьвает, что $A$ и $B$ не могут одновременно иметь нетривиальные полные части.

Предположим теперь, что $\widetilde{A} \neq 1$ и, значит, $\widetilde{B}=1$ и $B$ слойно конечна. Пусть $P-$ нетривиальная силовская подгрупша из $\widetilde{A}$ и пусть $b$ - элемент из $B \backslash A$. Ясно, что индекс $\left|B: C_{B}(b)\right|$ конечен; значит, пересечение $M=C_{D}(P) \cap C_{D}(b)$ бесконечно и $N_{G}(M)$ обладает обобщенно черниковской периодической частью $N$. Как и выше, мы получим, что $N$ содержится в $A$; следовательно, $b \in N \leqslant A$. Противоречие. Таким образом, $\widetilde{A}=\widetilde{B}=1$ и группы $A$ и $B$ обе слойно конечны.

Пусть $a \in A \backslash B$ и $b \in B \backslash A$. Тогда $C_{D}(\langle a, b\rangle)$ имеет конечньй индекс в $D$ и поэтому бесконечен. Пусть $d \neq 1$ - элемент из $C_{D}(\langle a, b\rangle)$. Обозначим через $C$ периодическую часть группы $C_{G}(d)$. Ясно, что $C$ содержится в $N_{G}((d))$ и, следовательно, является обобщенно черниковской группой. Более того, $E=A \cap C$ имеет конечный индекс в $A$; значит, пересечение $E$ бесконечно. Так как $C$ не лежит в $A$, то, как следует из первой части доказательства, $\widetilde{C}=1$, и поэтому группа $C$ слойно конечна. В частности, $(b)^{C}-$ конечная группа и $E$ имеет конечный индекс в $C_{1}=E(b)^{C}$. Используя предложение 8 из [11] видим, что $E$ содержит подгруппу конечного индекса $U$, которая нормализуется подгруппами $A$ и $C_{1}$. С другой стороны, $U$ слойно конечна; значит, она содержит конечную нетривиальную характеристическую подгруппу и $N_{G}(U)$ обладает обобщенно черниковской периодической частью. Так как $A<\langle A, b\rangle \leqslant N_{G}(U)$, мы получили противоречие. Лемма доказана.

По теореме из [12] группа $G$ содержит элемент $a \neq 1$ такой, что $C_{G}(a)$ не обладает черниковской периодической частью. Ясно, что периодическая часть $N$ группы $N_{G}((a))$ является обобщенно черниковской группой, и по лемме 4 мы можем рассмотреть максимальную обобщенно черниковскую подгруппу $H$ из $G$, содержащую $N$. Зафиксируем за этой подгруппой обозначение $H$ до конца доказательства теоремы 1.

Заметим, что ввиду выбора элемента $а$ группа $H$ не является черниковской.

Лемма 6. Пусть $b$-неединичный элемент из $H$ такой, что $C_{H}(b)$ бесконечен. Тогда периодическая часть иентрализатора $C_{G}(b)$ содержится в $H$.

ДокАЗАТЕльство. Пусть $M$ - максимальная обобщенно черниковская подгруппа из $G$, содержащая периодическую часть группы $C_{G}(b)$. Тогда пересечение $H \cap M$ бесконечно по условию леммы и $H=M$ по лемме 5. Таким образом, периодическая часть централизатора $C_{G}(b)$ содержится в $H$. Лемма доказана.

Лемма 7. Все әлементы простых порядков из Н имеют бесконечные чентрализаторы в $H$. 
ДокАЗАТЕЛЬСТво. Предположим, что подгрупша $H$ содержит такой элемент $b$ простого порядка, что $C_{H}(b)$ конечен. Тогда централизатор нормального замыкания $\left\langle b^{H}\right\rangle$ в группе $H$ элемента $b$ также конечен. Ввиду следствия 2 Половицкого из [13] фактор-группа $H / C_{H}\left(\left\langle b^{H}\right\rangle\right)$ черниковская. Таким образом, группа $H$ должна быть черниковской как расширение конечной группы при помоши черниковской группы. Противоречие. Лемма доказана.

4. Перейдем непосредственно к доказательству теоремы 1. Необходимость условий теоремы очевидна. Докажем их достаточность. Предположим, что $G-\operatorname{coпряженно~би-~}$ примитивно конечная группа без инволюций, не обладающая обобщенно черниковской периодической частью, и для каждой нетривиальной конечной подгрупшы $X$ из $G$ ее нормализатор $N_{G}(X)$ обладает обобщенно черниковской периодической частью.

По лемме 7 все элементы простых порядков из $H$ имеют бесконечные централизаторы в $H$. Обозначим через $M$ нормализатор подгруппы $H$ в группе $G$. Из строения обобщенно черниковских групп и условий теоремы вытекает, что группа $M$ обладает обобщенно черниковской периодической частью (ввиду максимальности $H$ эта периодическая часть совпадает с $H)$.

Пусть $g$ - элемент из $G \backslash M$, и предположим, что $H \cap g^{-1} H g \neq 1$. Если $b$ - элемент простого порядка из $H \cap g^{-1} H g$, то централизатор $C_{H}(b)$ бесконечен, и поэтому его периодическая часть содержится в $H$ по лемме 6 . С другой стороны, $b$ имеет бесконечньй централизатор еще и в $g^{-1} H g$; следовательно, периодическая часть $C_{G}(b)$ содержится также и в $g^{-1} H g$. Тогда пересечение $H \cap g^{-1} H g$ бесконечно и, поэтому $g^{-1} \mathrm{Hg}=H$ по лемме 5. Но это противоречит выбору элемента $g$ из $G \backslash M$. Следовательно, $H \cap g^{-1} H g=1$ и пересечение $M \cap g^{-1} M g$ не содержит неединичных элементов конечного порядка для любого элемента $g \in G \backslash M$. Тогда в конечной групше $\left\langle a, a^{g}\right\rangle$ (она конечна ввиду сопряженно бипримитивной конечности группы $G$ ) собственная подгруппа $\left\langle a, a^{g}\right\rangle \cap H$ составляет с $H$ пару Фробениуса и сама $\left\langle a, a^{g}\right\rangle$ является группой $\Phi$ робениуса по известной теореме Фробениуса. По теореме из [14] либо $G=F \lambda N_{G}((a))$ и $G=F \lambda(a)$ - группа робениуса, либо элемент $a$ содержится в конечном нормальном делителе групшы $G$. Вторая альтернатива невозможна ввиду предположения. Значит, $G=F \lambda(a)$ - группа $\Phi$ робениуса. Применяя теорему из [15], получаем, что $F-$ периодическая группа. Теперь при помощи леммы 4.6 из [16] приходим к противоречию с единичностью локально конечного радикала группы $G$ (он должен быть единичньпм при сделанных предположениях по лемме 3). Это последнее противоречие завершает доказательство теоремы 1.

Теперь чтобы доказать теорему 2 , нам достаточно сослаться на следующую теорему.

ТЕоремА. (Сенашов В.И. [1]) Пусть $G$ - группа с инволюииями, удовлетворяюиая условиям:

1) любые две инволюиии из $G$ порождают конечную подгруппу;

2) нормализатор любой конечной нетривиальной подгруппы, содержащий инволюиии, обладает обобщенно черниковской периодической частью.

Тогда либо $G$ обладает обобщенно черниковской периодической частью, либо G-T-гpynna.

Действительно, необходимость условий теоремы 2 очевидна. Докажем их достаточность. Предположим, что $G$ - сопряженно бипримитивно конечная группа, не обладающая обобщенно черниковской периодической частью, и для каждой нетривиальной ко- 
нечной подгрупшы $X$ из $G$ ее нормализатор $N_{G}(X)$ обладает обобшенно черниковской периодической частью.

Ввиду теоремы 1 группа $G$ обладает инволюциями. В то же время $T$-группа по определению (см., например, [1]) не может быть сопряженно бипримитивно конечной. Теперь, сославшись на приведенную выше теорему, получаем справедливость утверждения теоремы 2 .

\section{СПИСОК ЦИТИРОВАННОЙ ЛИТЕРАТУРЫ}

[1] Сенашов В.И. Характеризация обобщенно черниковских групп в группах с инволюциями // Матем. заметки. 1997. Т. 62. №4. С. 577-587.

[2] Сенашов В.И. Характеризация обобщенно черниковских групп // Докл. РАН. 1997. T. 352. №3. C. 309-310.

[3] Шунков В. П., Шафиро А. А. Об одной характеризации обобщенно черниковских групп // Материалы 15 Всесоюзн. алгебраич. конф. Красноярск: Красноярский государственный университет, 1979. С. 185.

[4] Половицкий Я.Д. Слойно-экстремальные группы // Матем. сб. 1962. Т. 56. №1. C. $95-106$.

[5] Адян С. И. Проблема Бернсайда и тождества в группах. М.: Наука, 1975.

[6] Ольшанский А. Ю. Геометрия определяюших соотношений в группе. М.: Наука, 1989.

[7] Остыловский А.Н. Локальная конечность некоторых групп с условием минимальности для абелевых подгрупп // Алгебра и логика. 1977. Т. 16. №12. С. 63-73.

[8] Каргаполов М.И. Локально конечные группы, обладающие нормальными системами с конечными факторами // Сиб. матем. ж. 1961. Т. 2. №6. С. 853-873.

[9] Ivko M. N., Senashov V. I. On a new class of infinite groups // Укр. матем. ж. 1995. T. 46. № 6. C. $760-770$.

[10] Сенашов В. И. Слойно конечные группы. Новосибирск: Наука, 1993.

[11] Шунков В.П. О локально конечных группах конечного ранга // Алгебра и логика. 1971. T. 10. № 12 . C. 199-225.

[12] Сучкова Н. Г. О существовании периодической части в некоторых бесконечных группах // Тезисы докладов Междун. конф. (Санкт-Петербург, Россия, 24-30 июня 1997 г.). С. 288.

[13] Половицкий Я. Д. О локально экстремальных и слойно-экстремальных группах // Матем. сб. 1962 . Т. 58. № 2. С. 685-694.

[14] Созутов А. И., Шунков В. П. Об одном обобщении теоремы Фробениуса на бесконечные группы // Матем. сб. 1976. Т. 100. №4. С. 495-508.

[15] Созутов А.И., Шунков В.П. О бесконечных группах, насыщенных фробениусовыми подгруппами. 2 // Алгебра и логика. 1979. Т. 18. № 2. С. 206-223.

[16] Шунков В. П. О вложении примарных элементов в группе. Новосибирск: Наука, 1992.

Институт вычислительной математики СО РАН,

Поступило г. Красноярск 